\title{
Factors Associated With Poor Functional Outcome Despite Low Post- treatment Infarct Volume in the ESCAPE Randomized Controlled Trial
}

Aravind Ganesh, Bijoy K. Menon, Zarina Assis, Andrew M. Demchuk, Fahad S Al-Ajlan, Jeremy L. Rempel, Blaise W. Baxter, John Thornton, Nima Kashani, Michael D. Hill, Mayank Goyal, for the ESCAPE Trialists

Background and Aims Improved outcomes after endovascular thrombectomy (EVT) are associated with smaller infarcts. However, patients with small post-treatment infarct volumes (PIV) sometimes fare poorly. We explored factors potentially associated with low PIV and poor outcome, hypothesizing differences in comorbidities and/or post-stroke complications.

Methods: We identified ESCAPE patients (EVT/control) with $\mathrm{PIV} \leq 25^{\text {th }}$ percentile, per 24-48-hour CT/MRI. We compared "discrepant cases"- 90-day modified Rankin Scale(mRS) score $\geq 3$ despite low PIV - against "non-discrepant cases"(mRS $0-2$ ), examining imaging modality, other outcomes (Barthel Index[BI], EQ-5D), ASPECTS locations, NIHSS, post-stroke complications, demographics, and comorbidities using Fisher's exact for binary variables and Wilcoxon rank-sum for continuous variables. Multi-variable logistic regression was used to evaluate factors independently associated with low-PIV/poor-outcome.

Results: Median PIV was $21 \mathrm{~mL}$ (IQR 7-72mL, $\mathrm{n}=315$ ). 79 had $\mathrm{PIV} \leq 7 \mathrm{~mL} ; 27(34.2 \%)$ were discrepant cases(mRS $\geq 3$ ).

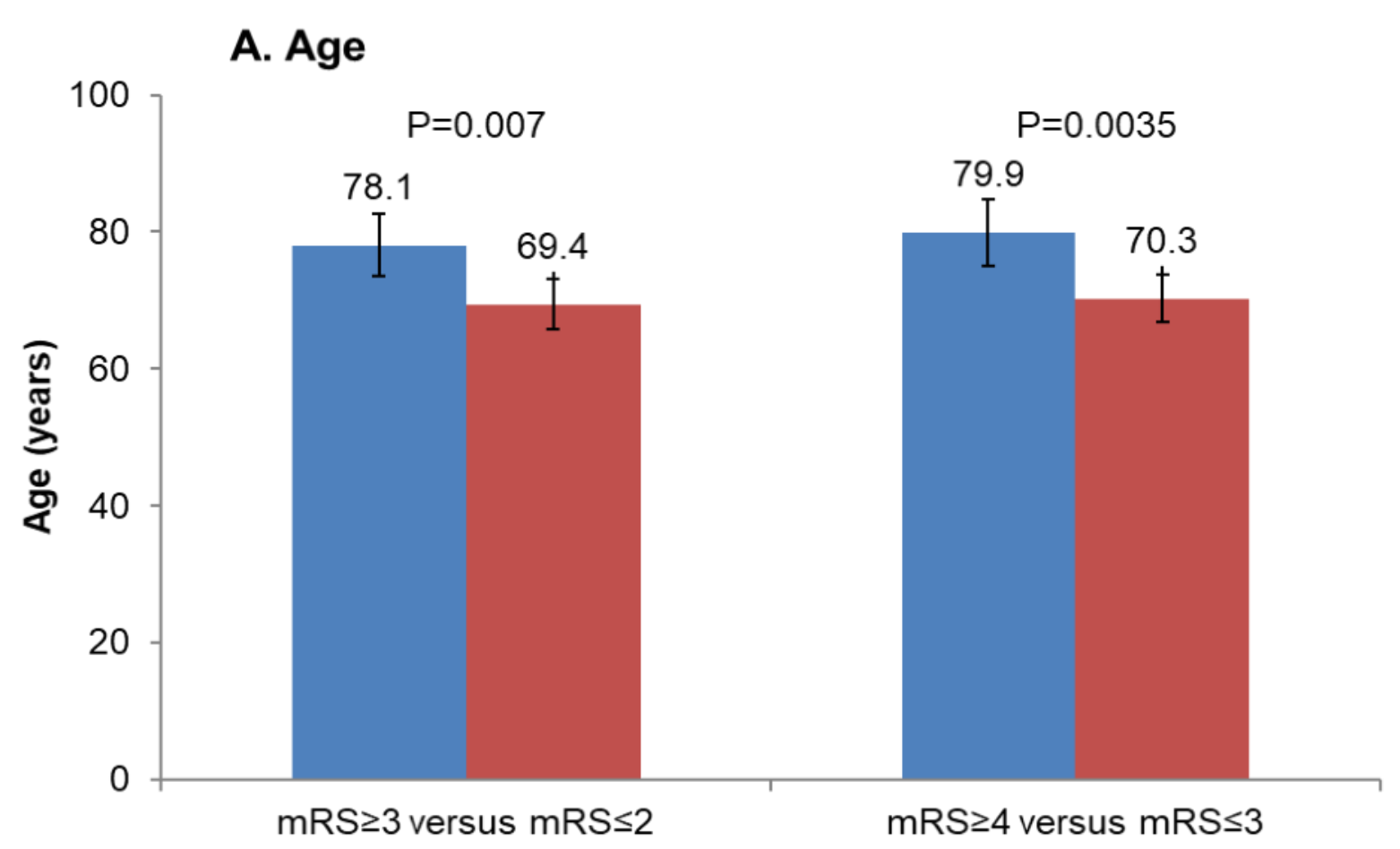

C. 24-hour NIHSS

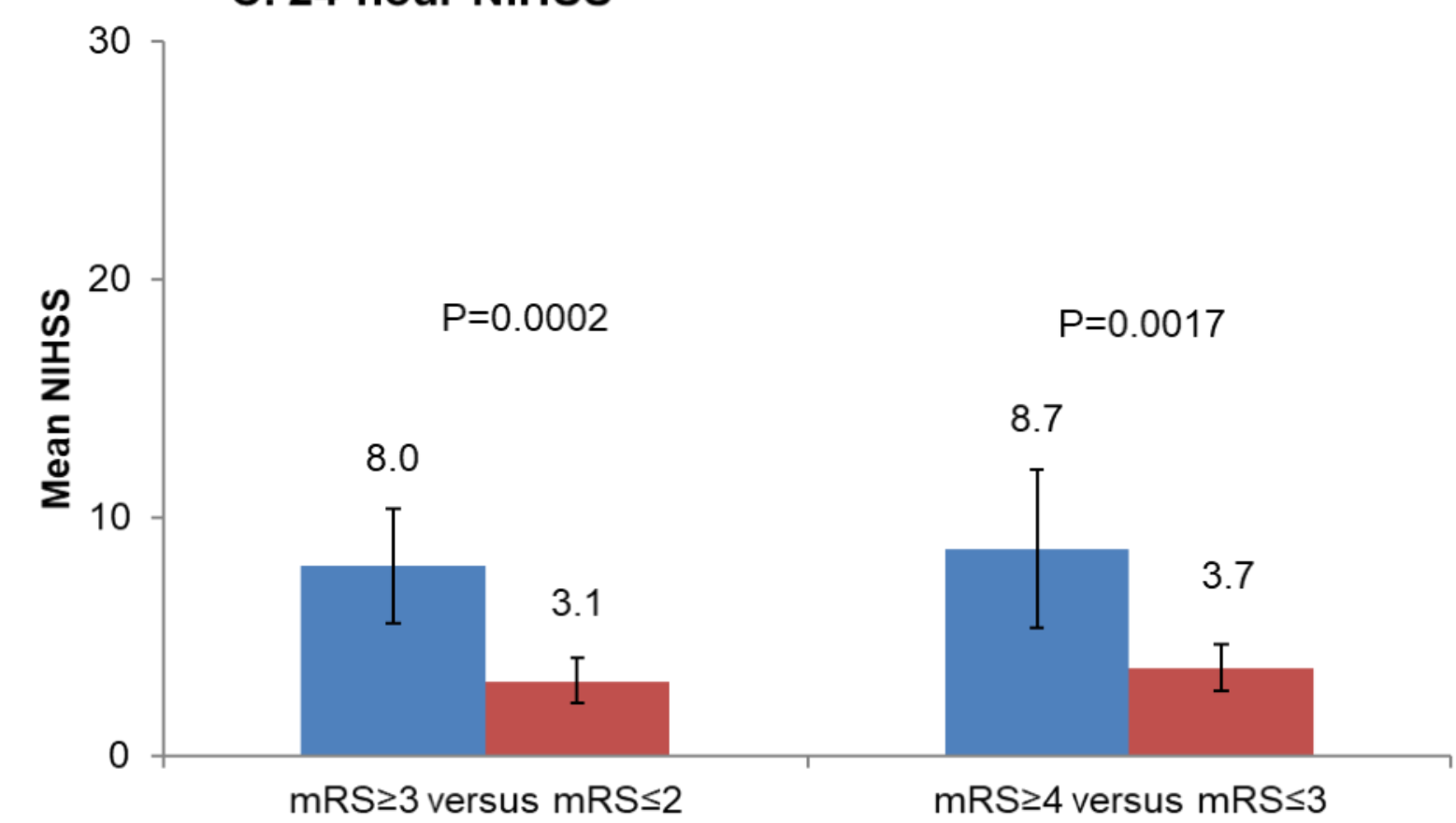

Compared to non-discrepant cases with PIV $\leq 7 \mathrm{~mL}$, discrepant cases had worse 90-day $\mathrm{BI}$ and EQ-5D (e.g. mean $\mathrm{BI} 44.6$, $95 \% \mathrm{Cl} 27.1-62.1$ vs 98.9,97.4-99.9,p<0.001), with no significant differences in EVT use (53.9\% vs $69.2 \%, \mathrm{p}=0.22)$ or CT versus $\mathrm{MRI}$ for volume ascertainment (84.6\% vs $75.0 \%, \mathrm{p}=0.39)$. However, discrepant cases had higher 24-hour/48-hour NIHSS (mean 24-hour 8.0,5.6-10.4 vs 3.1,2.2-4.1, $\mathrm{p}=0.0002$ ), were older (mean age $78.1,73.5-82.7$ vs $69.4,65.7-73.2, p=0.007$ ), more frequently had cancer $(15.4 \%$ vs $0 \%, p=0.01)$, and post-stroke complications $(42.3 \%$ vs $5.7 \%, \mathrm{p}<0.0001)$, especially pneumonia $(11.5 \%$ vs $0 \%, \mathrm{p}=0.03)$. On multi-variable regression, age $(p=0.025), \quad 24-/ 48$-hour $\operatorname{NIHSS}(p=0.015)$, post-stroke complications $(p=0.007)$ or cancer(perfect) were independently associated with low-PIV/poor-outcome.

Conclusions: Poor outcome despite low PIV is not explained by discrepancies in imaging modality or outcome assessment, but is associated with older age, 24-/48-hour NIHSS, comorbidities (cancer), and post-stroke complications. Preventing complications may mitigate discrepant outcomes.
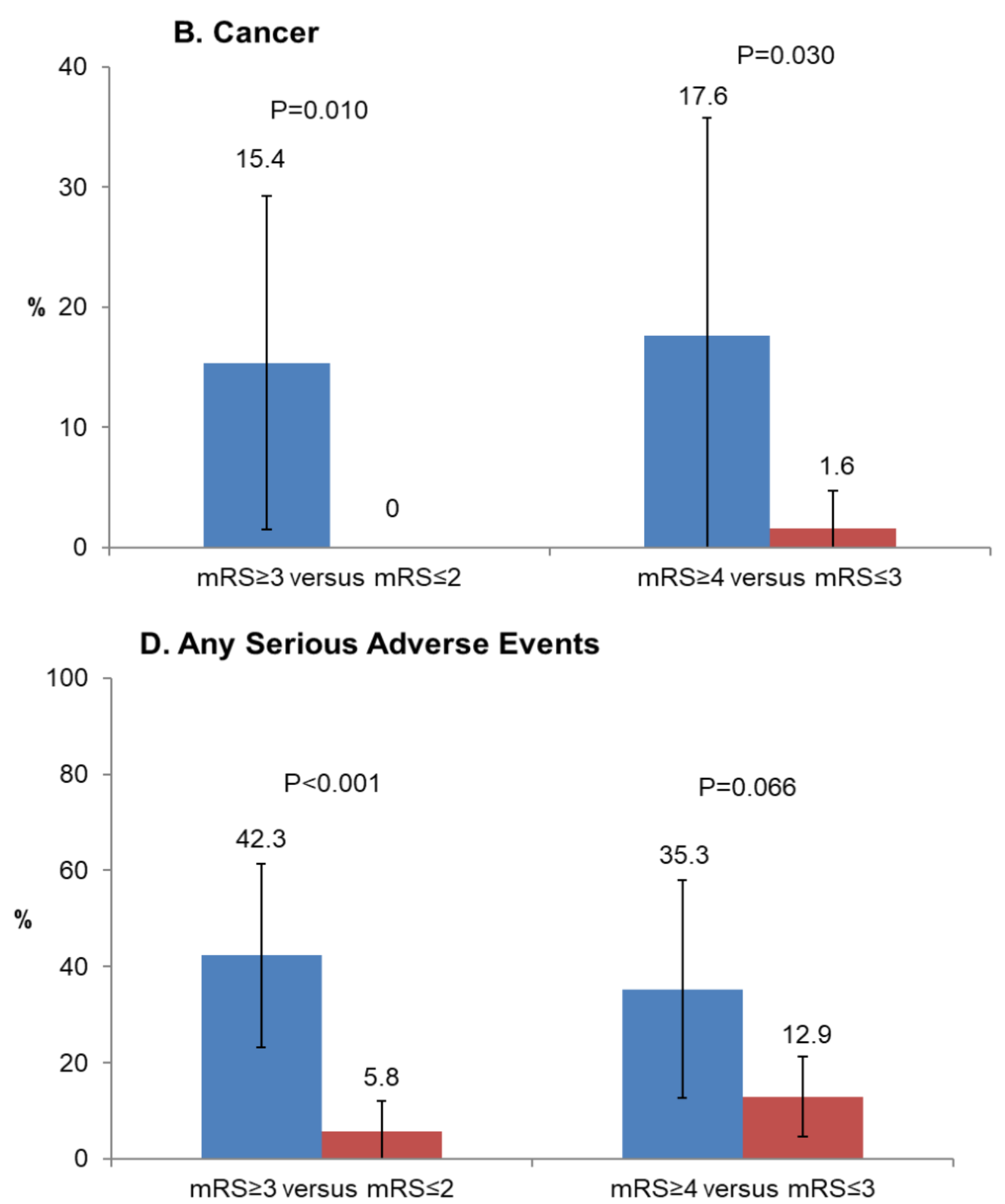

Figure 1 - Comparison of representative key variables that differed between patients with small post-treatment infarct volume (PIV $\leq 7 \mathrm{~mL}$ ) and poor outcome ("discrepant", blue, defined as either $\mathrm{mRS} \geq 3$ or $\mathrm{mRS} \geq 4$ ) and those with PIV $\leq 7 \mathrm{~mL}$ and good outcome ("non-discrepant", red): (A) age, (B) cancer, (C) 24-hour NIHSS, and (D) post-stroke serious adverse events (SAEs). 